\title{
Responsabilidad Social Empresarial e Innovación como Estrategia Competitiva en el Sector Turístico de Ciudad del Carmen, Campeche
}

\section{Corporate Social Responsibility and Innovation as a Competitive Strategy in the Tourist Sector of Ciudad del Carmen, Campeche}

\author{
Coliaza Palomino María Fernanda*, López Noriega Myrna Delfina**
}

*Estudiante de Ingeniería del Instituto de la Región Sierra de Teapa, Tabasco. Email: mary_jokers@hotmail.com, ORCID: https://orcid.org/0000-0003-4030-2073.

**Arquitecta de la Universidad Autónoma de Guadalajara, Maestra en Arquitectura por la Universidad Autónoma de Guadalajara, Maestra en Administración por la Universidad La Salle, Máster en Economía y Organización de Empresas por la Universidad de Granada, España, Doctora en Administración por la Universidad del Sur. Profesor Investigador de la Universidad Autónoma del Carmen, Cd. del Carmen, Campeche, México. Email: mdlopez@pampano.unacar.mx, ORCID: https://orcid.org/0000-0003-14268406.

Correo para recibir correspondencia: mdlopez@pampano.unacar.mx 
RESPONSABILIDAD SOCIAL EMPRESARIAL E INNOVACIÓN COMO ESTRATEGIA COMPETITIVA EN EL SECTOR TURÍSTICO DE CIUDAD DEL CARMEN, CAMPECHE.

\section{RESUMEN}

OBJETIVO: Identificar los factores de responsabilidad social e innovación empresarial que se presentan en las pequeñas y medianas empresas (pymes) del sector turismo de Ciudad del Carmen, Campeche.

MATERIAL Y MÉTODO: La metodología usada para el estudio es cuantitativa no experimental de corte transversal descriptiva y de método deductivo. Para la obtención de datos, se aplicó una encuesta a 119 empresas del sector turismo de dicha localidad.

RESULTADOS: El análisis de los datos recabados arrojó que la dimensión ambiental de la RSE es la menos valorada para los empresarios del sector turístico de Ciudad del Carmen, Campeche.

CONCLUSIONES: Las empresas actualmente se encuentran en un mercado caracterizado por grandes cambios, resultado de la globalización; es decir, que existe un sinfín de empresas ofreciendo el mismo producto o servicio. En ese contexto, es de suma importancia que las organizaciones identifiquen estrategias competitivas que le permitan permanecer y subsistir a las exigencias del consumidor.

PALABRAS CLAVE: Responsabilidad social. Estrategia competitiva. Globalización. Innovación. Dimensiones de RSE.

\section{ABSTRACT}

OBJECTIVE: Identify the factors of social responsibility and business innovation that occur in small and medium-sized enterprises (SMEs) in the tourism sector of Ciudad del Carmen, Campeche.

MATERIAL AND METHOD: The methodology used for the study is quantitative, non-experimental, descriptive cross-sectional and deductive method. To obtain data, a survey was applied to 119 companies in the tourism sector in that town.

RESULTS: The analysis of the data collected showed that the environmental dimension of CSR is the least valued for entrepreneurs in the tourism sector of Ciudad del Carmen, Campeche. 
RESPONSABILIDAD SOCIAL EMPRESARIAL E INNOVACIÓN COMO ESTRATEGIA COMPETITIVA EN EL SECTOR TURÍSTICO DE CIUDAD DEL CARMEN, CAMPECHE.

CONCLUSIONS: Companies are currently in a market characterized by great changes, result of globalization; In other words, there are countless companies offering the same product or service. In this context, it is of utmost importance that organizations identify competitive strategies that allow them to remain and survive the demands of the consumer.

KEY WORDS: Social responsibility. Competitive strategy. Globalization. Innovation. CSR dimensions.

\section{INTRODUCCIÓN}

La presente investigación aborda el tema de Responsabilidad Social Empresarial (RSE) que se puede definir, según Bowen (1953), como "las obligaciones de los hombres de empresa a seguir aquellas políticas, tomar aquellas decisiones, o seguir las líneas de acción que resulten deseables en función de objetivos y valores de nuestra sociedad"; de igual forma, se abordará el tema de innovación empresarial, considerado como un proceso de construcción creativa, que permite que la economía y los agentes económicos evolucionen, por lo que es la forma en que la empresa administra sus recursos a través del tiempo y desarrolla competencias que influyen en su competitividad (Schumpeter, 1934). Ambos temas son estrategias que, en un mercado de gran cambio, las empresas han utilizado como creadores de ventajas competitivas, ante consumidores que esperan más de ellas y les exigen que jueguen un papel importante en el desarrollo y crecimiento de la calidad de vida de los trabajadores, de la comunidad y del país.

El turismo representa el sector económico más grande de México, al tener la mayor aportación al $\mathrm{PIB}$, y ocupa, al mismo tiempo, el segundo lugar en la creación de empleos entre las industrias que proveen trabajos en el país (World Travel \& Tourism Council, 2016). Adicionalmente, en una economía caracterizada por pequeñas y medianas empresas (pymes) que generan el $72 \%$ del empleo y el 52\% del Producto Interno Bruto (PIB), conforme con los Censos Económicos del Instituto Nacional de Estadística y Geografía (INEGI) (INEGI, 2014), las pymes del sector turismo mexicano representan parte importante de ese sector. 
RESPONSABILIDAD SOCIAL EMPRESARIAL E INNOVACIÓN COMO ESTRATEGIA COMPETITIVA EN EL SECTOR TURÍSTICO DE CIUDAD DEL CARMEN, CAMPECHE.

Pese a lo anterior, las pymes mexicanas presentan bajo crecimiento y poca escalabilidad en el país, con una productividad 2.9 y 1.7 veces menor con respecto a las grandes (OCDE, 2019). En ese sentido, la RSE y la innovación en las pymes del sector turismo, representan la posibilidad de permanecer en un mercado nacional altamente competitivo.

Así, el objetivo general de este trabajo es describir los elementos de RSE e innovación empresarial que se presentan en las pymes del sector turismo de Ciudad del Carmen, Campeche, como parte importante de la economía local y nacional. Para lograr lo anterior, se incorpora un amplio campo de estudio en materia de dos variables actuales: la RSE y la innovación a partir de sus teorías y el análisis de los beneficios que una empresa obtiene al aplicar sus estrategias. Bajo esa idea, se aplicó una encuesta a partir de la propuesta de Gallardo-Vázquez y SánchezHernández (2013) a una muestra, no probabilística por conveniencia, de 119 empresarios y dueños de este tipo de negocios en Ciudad del Carmen, Campeche.

\section{REVISIÓN LITERARIA}

Duque-Orozco et al. (2013) hicieron un recorrido por los conceptos de la RSE, a partir de los enfoques y modelos de autores como: Bowen (1953), McGuire (1963), Davis (1967), Friedman (1970), Drucker (1984), Wood (1991), Carroll (1991), Porter y Kramer (2006). También, realizaron una aproximación a las normas y guías elaboradas por diversos organismos como la Comisión de Comunidades Europeas (CCE, 2001), El Pacto Mundial, Global Reportiting Initiative (GRI, s.f.), SA 8000, International Organization for Standardization (ISO, 2010) e Instituto Ethos (2005).

Como resultado, Duque-Orozco et al. (2013) concluyeron que, existe la necesidad de originar consensos frente a las normas y auditorías de RSE de tal manera que esta sea regulada en lo más exiguo deseable por las sociedades, sin olvidar que la RSE debe ser de carácter voluntario, de lo contrario se comprometería su existencia, logrando que se perdiera la esencia de la RSE.

Por otra parte, Gallardo-Vázquez y Sánchez-Hernández (2013) analizaron de manera empírica a través de la técnica de ecuaciones estructurales la incidencia de la RSE en el éxito competitivo de los negocios que caracteriza el tejido productivo de 710 microempresas de Extremadura, España. Con ello, identificaron la relación de la RSE y la competitividad empresarial, y la innovación, como aspectos importantes de la gestión y dirección de empresas. Los resultados confirmaron que, un enfoque de gestión responsable incide de forma determinante en la 
RESPONSABILIDAD SOCIAL EMPRESARIAL E INNOVACIÓN COMO ESTRATEGIA COMPETITIVA EN EL SECTOR TURÍSTICO DE CIUDAD DEL CARMEN, CAMPECHE.

competitividad de esas empresas, al igual, existe una relación entre la innovación y la RSE, con un efecto parcial, pero con resultados positivos significativos.

Macías (2012), en su investigación “Innovación y responsabilidad social: Una reflexión sobre el punto de encuentro", propone relacionar la innovación con la RSE, en la búsqueda de efectos de la innovación diferentes a los resultados económicos y la sostenibilidad de las empresas en el mediano plazo; el investigador se interesa particularmente por encontrar los impactos sociales de la innovación, que es el fin último de la RSE.

Para lograr lo anterior, Macías (2012) identifica cuatro vínculos entre la RSE y la innovación, entre los cuales están: la innovación disruptiva, la cual consiste en ofrecer productos que no tienen características similares a los productos de los mercados maduros, no obstante, se adaptan a las mismas necesidades de un público menos exigente, que comúnmente son los consumidores de bajo ingreso a los cuales Porter y Kramer (2002) habían aludido; innovación organizacional, la cual se basa en el nivel de innovación que existe en las organizaciones que cada vez se enfoca en dar un beneficio a la sociedad; la relación entre la innovación y el emprendimiento, que consiste en el emprendimiento con el fin de ayudar a la sociedad a través de la innovación; y, por último, la innovación social que está integrada de emprendedores sociales que buscan dar solución a los mayores problemas de la sociedad. Así, los vínculos que el autor plantea entre la innovación y la RSE parten del beneficio social y, al mismo tiempo, económico hacia las organizaciones.

\section{MARCO TEÓRICO}

\section{Concepto de RSE}

Para comprender el alcance de la RSE, es necesario considerar su conceptualización, de ahí que se considere el concepto introducido por Bowen a mediados del siglo $\mathrm{XX}$, quien en ese tiempo pensaba que las empresas eran vitales y que las decisiones de estas tenían repercusiones de igual forma en la sociedad. Bowen (1953) menciona que la RSE son "las obligaciones de los hombres de empresa a seguir aquellas políticas, tomar aquellas decisiones, o seguir las líneas de acción que resulten deseables en función de objetivos y valores de nuestra sociedad" (p. 6). Mientras que, Friedman (1962) señala que la actividad vital de la Responsabilidad Social (RS) de 
RESPONSABILIDAD SOCIAL EMPRESARIAL E INNOVACIÓN COMO ESTRATEGIA COMPETITIVA EN EL SECTOR TURÍSTICO DE CIUDAD DEL CARMEN, CAMPECHE.

la empresa es la de maximizar la riqueza para el accionista, cuando se encuadre dentro del marco legal a que éstas se encuentran sujetas y sin ser objeto de una conducta fraudulenta.

Davis (1973) relacionó a la RSE con la consideración y respuesta de la empresa a cuestiones que van más allá de temas estrictamente económicos, técnicos o legales, por lo que señaló que es la obligación de una empresa de evaluar los efectos de sus decisiones sobre el sistema social externo a modo de lograr beneficios sociales además de las ganancias económicas tradicionales buscadas. Finalmente, Garriga y Melé (2004) agruparon la RSE bajo cuatro grupos de teorías, conforme con el enfoque del autor, dentro de las que destaca el que aborda las prácticas de RSE como herramientas estratégicas que permite lograr mejores beneficios empresariales, y el crecimiento de la organización como el grupo de las teorías integradoras que se basan, principalmente, en la satisfacción de las demandas o exigencias de los stakeholders.

\section{La RSE como parte de la estrategia empresarial}

En sus inicios, la RSE aparece relacionada con actividades filantrópicas; sin embargo, las exigencias del mercado cambiaron el enfoque a finales de los años ochenta, cuando la orientación filantrópica y de cumplimiento legal evolucionó a una visión estratégica y relacionada con la competitividad (Porter y Karmer, 2002); de ahí que Schroeder y Kilian (2007) señalan que ninguna empresa realiza actividades al margen del negocio, sin ninguna connotación estratégica. Por lo tanto, la RSE está relacionada con la competitividad, ya que realiza una conexión entre el beneficio que la empresa obtendrá al aplicarla y los beneficios sociales que surgirán. La RS como ventaja competitiva, conforme a Schroeder y Kilian (2007) "involucra un pensamiento estratégico, vinculado a las oportunidades de ganar u obtener beneficios más elevados, ya sea vía mejoras en la reputación, fortalecimiento de las marcas, o a través de la innovación en productos, servicios y procesos" (p. 5).

Adicionalmente, las organizaciones se encuentran en un mercado globalizado en donde las exigencias de los clientes son cada vez mayores y la competencia es constante, por lo que surge la necesidad de crear nuevas estrategias para conseguir un mejor nivel de competitividad. En ese contexto, la RSE se enfoca en conectar a las empresas con el entorno y, al mismo tiempo, que éstas obtengan múltiples beneficios competitivos respecto a las demás. Así, según Toro (2006), se concibe que la RSE está vinculada con la visión, misión y valores que la empresa ha 
RESPONSABILIDAD SOCIAL EMPRESARIAL E INNOVACIÓN COMO ESTRATEGIA COMPETITIVA EN EL SECTOR TURÍSTICO DE CIUDAD DEL CARMEN, CAMPECHE.

desarrollado. De igual manera, la RSE como estrategia fija el posicionamiento de la organización y permite alcanzar sus metas a largo plazo y mantenerse competitiva.

Otro rasgo de la importancia de la RSE radica en gran medida en los efectos positivos que deja en la organización como lo es un mejor posicionamiento de marca y, a su vez, una reputación impecable. De lo anterior, se puede inferir que la visión actual de la RSE tiene un fin estratégico que está vinculado con la competitividad empresarial, y dicho comportamiento implica, a decir de Bermúdez (2007), la mejora de la imagen impactando positivamente en la competitividad de la empresa. De manera que la RSE, además de ser una estrategia competitiva, también juega un papel importante ante stakeholders cada vez más exigentes que demandan que las empresas respondan por los impactos negativos que generan en el medio ambiente o en la sociedad, obligando a que intenten disminuirlos.

A su vez, los administradores implementan prácticas de RSE de acuerdo con los beneficios esperados, con las características de la empresa, con el sector en el cual se ubican y dependiendo de elementos como misión, valores, liderazgo, cultura interna o estrategia de negocios y, de acuerdo con Flores et al. (2007), entre los efectos positivos de la RSE se encuentran los estímulos para la innovación.

\section{La innovación como parte de la ventaja competitiva}

El entorno donde se encuentran las empresas ha cambiado a lo largo del tiempo a un ritmo acelerado; hoy en día, las ventajas que el empresario adopta son de carácter pasajero, por lo que la empresa con mejores ventajas competitivas es la que permanece a flote. De esta manera, surgen interrogantes sobre cuál es la mejor manera de mantener el crecimiento de la empresa, a la que diversos autores han respondido que hay que identificar las ventajas competitivas de largo plazo, pero con resultados seguros (Porter y Kramer, 2002), es decir, la ventaja sostenible. En ese sentido, se ha concluido que desarrollar continuamente la innovación proporciona mejores ventajas competitivas y sostenibles a largo plazo.

Porter y Kramer (2002) señalan que dentro del conjunto de estrategias para lograr ventajas competitivas se encuentran las de la base de la pirámide económica, en la que varios autores ven una oportunidad para innovar. Sin embargo, innovar de tal forma que sea productivo es más difícil para las empresas, no obstante, las organizaciones están dispuestas a asumirla esperando 
RESPONSABILIDAD SOCIAL EMPRESARIAL E INNOVACIÓN COMO ESTRATEGIA COMPETITIVA EN EL SECTOR TURÍSTICO DE CIUDAD DEL CARMEN, CAMPECHE.

garantías a largo plazo. Hidalgo et al. (2002) declaran que la capacidad de innovar constituye un recurso de la empresa cada vez más valioso y, al igual que sus capacidades financieras, comerciales y productivas, debe ser gestionado de manera rigurosa y eficiente. De lo anterior, se concluye que la adecuada gestión de la innovación es vital para el crecimiento de las empresas.

Aunque está demostrado que el efecto de las Tecnologías de la Información y Comunicación (TIC) sobre el resto de la economía provoca que la innovación dinamice actividades que antes estaban ajenas a ello, la innovación no siempre se asocia con tecnología (Scarone, 2005), a tal grado que un producto puede ser muy novedoso, pero esto no siempre conlleva a que sea innovador, de ahí que la innovación está mal entendida. Además, como señala Scarone (2005), la innovación es un concepto complejo y su carácter abstracto, multi-dimensional y abarcativo, hace más difícil su conceptualización; este término, ha sido definido por varios autores, por lo que, de manera general, se puede decir que la innovación es un camino de conocimientos para llegar a un objetivo.

\section{La innovación}

Si bien, para Nieto y Fernández (2004) la RSE es, en sí misma, una innovación, es necesario partir de su definición; en este caso se ha partido de su concepción amplia, como "la adopción de una nueva idea o práctica que puede dar inicio a nuevos productos, mercados, procesos productivos, organizacionales o administrativos" (Gallardo-Vázquez y Sánchez-Hernández, 2013, p. 221), por lo que las autoras consideraron todas las facetas del concepto de innovación consideradas en el Manual de Oslo: de producto, de proceso, de organización y de mercadotecnia (OCDE, 2005 citado en Gallardo-Vázquez y Sánchez-Hernández, 2013).

Una innovación de producto es "la introducción de un bien o de un servicio nuevo, o significativamente mejorado, en cuanto a sus características o en cuanto al uso al que se destina" (OCDE, 2005, p. 58). Al hablar de innovación de proceso se refiera a cuando se introduce un nuevo, o que este ha sido mejorado en su proceso de producción o de distribución, lo que implica por lo regular cambios tecnológicos. Mientras que, una innovación de mercadotecnia se cumple cuando se aplica un nuevo método de comercialización que lleve a cambios significativos del diseño o el envasado de un producto, su posicionamiento, su promoción o su tarificación". 
RESPONSABILIDAD SOCIAL EMPRESARIAL E INNOVACIÓN COMO ESTRATEGIA COMPETITIVA EN EL SECTOR TURÍSTICO DE CIUDAD DEL CARMEN, CAMPECHE.

Finalmente, la innovación organizativa, como su nombre lo indica considera en un nuevo método organizativo en las prácticas, o la organización del lugar de trabajo, incluso en las relaciones que la empresa tiene con otras organizaciones (OCDE, 2005).

Se puede considerar a la innovación, de manera general, como un proceso que permite modificar o desarrollar nuevos productos y servicios, que son percibidos y aceptados por los clientes. De hecho, un sinónimo de innovación es el cambio, por lo que se pude señalar que, en la administración, la innovación aborda el proceso de organizar y dirigir los recursos de la organización (humanos, materiales, económicos) con la finalidad de mejorar la competitividad de esta.

\section{Dimensiones de Responsabilidad Social Empresarial}

La RSE conforme Dahlsrud (2008) está formada por cinco dimensiones: la dimensión ambiental, la dimensión social, la dimensión económica, la dimensión de los grupos de interés o stakeholders y la dimensión del voluntariado; no obstante, para efectos de esta investigación se contemplarán solamente las primeras tres.

Para Gallardo-Vázquez y Sánchez-Hernández (2013) la dimensión social está relacionada con aspectos de los trabajadores como igualdad, conciliación laboral y familiar, la comunicación y participación en la empresa o la salud laboral y a su vez con la sociedad, tales como la creación de empleo, la atención a la discapacidad o las relaciones con las organizaciones no gubernamentales.

Mientras que la dimensión ambiental recoge aspectos relacionados con la minimización de los impactos, el ahorro energético y la protección del entorno y por último la dimensión económica relacionada con los clientes, la calidad de los productos y servicios, la ampliación de mercados o el tratamiento de las quejas e incluye la gestión de proveedores, la estabilidad de las relaciones o la preferencia por lo local (Gallardo-Vázquez y Sánchez-Hernández, 2013).

\section{Dimensiones de RSE y la innovación}

Las dimensiones de RSE y la innovación tienden al mejoramiento de la empresa a través de nuevos procesos, productos o ideas. En ese sentido, la innovación empresarial se manifiesta como una de las formas de RSE, pues adopta una postura activa y responsable para hacer 
RESPONSABILIDAD SOCIAL EMPRESARIAL E INNOVACIÓN COMO ESTRATEGIA COMPETITIVA EN EL SECTOR TURÍSTICO DE CIUDAD DEL CARMEN, CAMPECHE.

negocios que garanticen un mayor desarrollo sostenible, en donde el crecimiento económico vaya en una misma dirección con el bienestar social y el uso responsable de los recursos naturales.

Dado lo anterior, se ha considerado la propuesta que hacen Gallardo-Vázquez et al. (2013) al relacionar la RSE con otras variables empresariales a las que aluden como de naturaleza estratégica, entre ellas, la innovación. Para ello, la escala que proponen define a la RSE a partir de sus tres dimensiones: económica, social y medioambiental que determinan la orientación de la RSE por parte del empresario (Gallardo-Vázquez et al., 2013). Aunque Gallardo-Vázquez et al., (2013) no definen esas dimensiones, para efectos de esta investigación se ha considerado la definición y clasificación que realiza Cajiga (2017) de ellas.

Dimensión económica: generación y distribución del valor agregado entre integrantes y accionistas, considerando no sólo las condiciones de mercado sino también la equidad y la justicia. Se presume que la organización genere utilidades y se mantenga viva y eficaz (sustentabilidad). Al mismo tiempo involucra la generación y distribución de bienes y servicios útiles y rentables para la comunidad, además de su aportación a la causa pública vía la contribución impositiva (Cajiga, 2017).

Dimensión social: La responsabilidad compartida y subsidiaria de inversionistas, directivos, colaboradores y proveedores para el cuidado y fomento de la calidad de vida en el trabajo y el desarrollo integral y pleno de todos ellos; considerando, también la realización de acciones y aportaciones propias y gremiales seleccionadas para contribuir con tiempo y recursos (Cajiga, 2017).

Dimensión ecológica: Comprende la responsabilidad total sobre las repercusiones ambientales de sus procesos, productos y subproductos; y, por lo tanto, la precaución de los impactos que causen o pudieran causar, sin descuidar, el compromiso ante acciones específicas para contribuir a la preservación y mejora de la herencia ecológica común para el bien de la humanidad actual y futura (Cajiga, 2017).

Gallardo-Vázquez et al. (2013) prueban a través de un modelo la relación causal de la RSE sobre la innovación, para lo cual consideran a Nieto y Fernández (2004) y López et al. (2007), quienes afirman que aquellas organizaciones que realizan acciones de RS tienden a ser más innovadoras. 
RESPONSABILIDAD SOCIAL EMPRESARIAL E INNOVACIÓN COMO ESTRATEGIA COMPETITIVA EN EL SECTOR TURÍSTICO DE CIUDAD DEL CARMEN, CAMPECHE.

Por otro lado, también mencionan tanto los resultados de Shapiro (1983) y Kirchhoff (2000) que enfatizan la relación entre rendimiento resultado de la RS y la innovación, como las innovaciones organizativas impactan en los empleados, de acuerdo con los resultados de Turban y Greening (1997). Gallardo-Vázquez et al. (2013) concluyen sobre la existencia de una influencia positiva y directa de la RSE en la innovación empresarial.

Por lo que, no es de sorprender que Gallardo-Vázquez y Sánchez-Hernández (2013), señalen que "la innovación contribuye en un $13 \%$ de la varianza del éxito competitivo" (p. 26). En otras palabras, si la organización pone en práctica la innovación generará estrategias para su reputación y a su vez modelos que permitan que se ejecuten actividades en cuanto a la RSE en beneficio de la organización, así como de sus empleados, clientes, proveedores y la sociedad como tal.

\section{Importancia de la RSE y la innovación en el sector turismo}

En México, el turismo es una actividad que ha significado, en los últimos años, una importante oportunidad de crecimiento y desarrollo. Hasta antes de la pandemia por Covid-19, esta actividad y los servicios recreativos representaban aproximadamente el 3\% del PIB; aunque en algunos estados son el soporte de la economía local, haciéndolos más vulnerables a sus cambios (Mercado y Palmerín, 2012). Al mismo tiempo, con un cliente cada vez más exigente en el turismo, la RSE contribuye a la creación de productos turísticos sostenibles y a la generación de valor para éstos.

Pérez-Aranda (2015), sostiene que los modelos de negocios turísticos que incorporan el desarrollo de la RSE como estrategia de competitividad y sostenibilidad son cada vez de mayor interés para el turista, generando una ventaja respecto a sus competidores que no la incorporan. Más aún, la innovación es otro aspecto que el sector debe acuñar, debido a que optimiza los recursos, mejora la calidad del servicio y es una fuente generadora de valor, ya que, a través de las creaciones o modificaciones de los procesos, se logra tener una gran ventaja competitiva.

En cuanto al sector turismo en Ciudad del Carmen, Campeche, México, es el reflejo de la actividad petrolera que prevalece en la localidad como resultado de la crisis por la que atraviesa esta la que otrora fuera una actividad muy dinámica. "El sector se caracteriza por una población flotante 
RESPONSABILIDAD SOCIAL EMPRESARIAL E INNOVACIÓN COMO ESTRATEGIA COMPETITIVA EN EL SECTOR TURÍSTICO DE CIUDAD DEL CARMEN, CAMPECHE.

de miles de trabajadores y su principal cliente es el trabajador emergente petrolero, por lo que el turismo se puede denominar de negocios" (López-Noriega et al., 2019).

Dado que la crisis petrolera ha frenado la derrama económica en el municipio y, por ende, del estado, se vislumbra en el turismo una oportunidad para repuntar la economía estatal; pese a ello, uno de los factores que limitan el desarrollo del turismo radica en que la oferta y la demanda de productos y servicios están adecuadas a la actividad petrolera, presentando problemas para el proceso de adaptación, entre los que destaca la falta de competitividad, especialmente entre los hoteles y restaurantes de Ciudad del Carmen (López-Noriega et al., 2019).

\section{MATERIAL Y MÉTODO}

Esta investigación se realizó mediante un enfoque cuantitativo, con un diseño no experimental, (Hernández et al., 2014), con una recolección de datos transversal (Pérez, 2012). Además, el alcance fue descriptivo (Behar, 2008).

\section{Población y muestra}

La población de esta investigación son las pymes del sector turismo de Ciudad del Carmen, Campeche, que, según la coordinación de turismo municipal de dicha localidad, eran un total de 182 empresas, conformadas por 66 hoteles, 49 restaurantes, 16 cafeterías, 23 marisquerías, 16 bares y 12 agencias de viajes en el periodo de septiembre a diciembre 2019. La muestra, no probabilístico por conveniencia, calculada con un nivel de confianza de 95\% y un 5\% de margen de error, fue de 124 empresas, sin embargo, para el análisis de los datos, sólo 119 observaciones fueron válidas, debido a que solo estas cumplían con las características para la investigación, por lo que el margen de error se incrementó a 5.3\%, porcentaje no significativo sobre la desviación en la opinión de la población total en función del error tipo I que se pretende mantener, considerando que existe un 95\% de probabilidades de acertar.

\section{Instrumento}

Para la recolección de datos, se aplicó una encuesta a los gerentes, directores y/o encargados de las empresas para conocer la perspectiva que tienen sobre el nivel de responsabilidad social e innovación en éstas. El instrumento, compuesto por 64 ítems, fue adaptado a partir de la propuesta de Gallardo-Vázquez y Sánchez-Hernández (2013), en el que la variable de RSE fue 
RESPONSABILIDAD SOCIAL EMPRESARIAL E INNOVACIÓN COMO ESTRATEGIA COMPETITIVA EN EL SECTOR TURÍSTICO DE CIUDAD DEL CARMEN, CAMPECHE.

considerada como una variable de segundo orden, evaluada a partir de las dimensiones social, económica y medioambiental; a su vez, la variable innovación fue evaluada con 13 ítems de acuerdo con sus facetas consideradas en el Manual de Oslo (OCDE, 2005).

Para medir cada ítem, se utilizó una escala de Likert con puntajes que van del 0 al 5, donde el 1 significa totalmente en desacuerdo, mientras que 5, correspondió a totalmente de acuerdo y, finalmente 0, no sabe o no aplica. Cabe señalar, que Gallardo-Vázquez y Sánchez-Hernández (2013) mencionan que los indicadores de cada dimensión proceden de otras escalas de medida que han sido analizadas y adaptadas a las empresas consideradas en su investigación. A su vez, estos autores han partido de la concepción amplia sobre la innovación considerada en el Manual de Oslo (OCDE, 2005).

Para determinar la confiabilidad del instrumento se realizó el análisis de confiabilidad mediante el coeficiente Alfa de Cronbach, cuyo resultado para cada dimensión de RSE y para la variable innovación se observa en la Tabla 1, con lo que se puede concluir que el valor de estas es altamente confiable ya que el coeficiente de Alfa se encuentra próximo a 1 (Frías, 2019).

Tabla 1

Análisis de confiabilidad del instrumento

\begin{tabular}{lccc}
\hline Variable & Coeficiente de Alfa Cronbach & No. de ítems & Confiabilidad \\
\hline Dimensión social de RSE & .967 & 15 & Alta \\
Dimensión económica de RSE & .958 & 11 & Alta \\
Dimensión ambiental de RSE & .943 & 14 & Alta \\
Innovación & .939 & 13 & Alta \\
Total & .985 & 53 & Alta \\
\hline
\end{tabular}

Fuente: Elaboración propia a partir del resultado.

\section{ANÁLISIS Y RESULTADOS}

\section{Análisis sociodemográfico}

A partir de la muestra de las 119 empresas analizadas, se obtuvo que los gerentes o empresarios de dicho sector eran $66.39 \%$ hombres y $33.61 \%$ mujeres al momento de la encuesta. Asimismo, su promedio de edad fue de 33 años con desviación estándar de \pm 6.98 años, es decir, de manera general son adultos jóvenes, lo que se confirma al observar que los datos tienen una distribución 
RESPONSABILIDAD SOCIAL EMPRESARIAL E INNOVACIÓN COMO ESTRATEGIA COMPETITIVA EN EL SECTOR TURÍSTICO DE CIUDAD DEL CARMEN, CAMPECHE.

asimétrica con sesgo positivo y de forma leptocúrtica. En cuanto a los años de vida de las empresas, estas tenían un promedio de 14 años en ese periodo y concentraban en promedio 24 empleados en su plantilla, con una desviación estándar de \pm 14.80 .

Asimismo, se obtuvo que el $53.78 \%$ de los encuestados estaban casados, $44.54 \%$ solteros y el $1.68 \%$ divorciados. Además, que un $64.71 \%$ dijeron ser originarios de Ciudad del Carmen y $35.29 \%$ señalaron ser foráneos. También se puede mencionar que solo un $62.18 \%$ de los empresarios de la muestra encuestada terminaron una licenciatura, mientras que el 31.93\% restante solo concluyeron la preparatoria.

En cuanto al tamaño de las empresas, destaca que un $75 \%$ de la muestra eran empresas pequeñas, mientras que el $25 \%$ restante son medianas. Con respecto a la estructura de la organización, el $68.07 \%$ fueron empresas familiares y el $31.93 \%$ de estructura no familiar.

\section{Dimensiones de la RSE e innovación}

Con respecto a las dimensiones de la RSE y la innovación, el criterio que se siguió para interpretar los resultados de la encuesta con la escala de Likert de 5 puntos fue, respuestas mayores o iguales a 3.5 son consideradas como aceptables y respuestas menores a 3.5 se consideraron como no aceptables. Ese rango se definió con el propósito de que aquellas dimensiones de la RSE e innovación de las diferentes empresas, cuyo valor fuera menor a 3.5 fueran consideradas como áreas de oportunidad para éstas.

En la Tabla 2, se muestran los resultados de las dos variables analizadas, considerando las dimensiones de la variable RSE como de segundo orden. La dimensión social obtuvo un promedio de 3.60 siendo la más alta calificación, sucesivamente la dimensión económica con un promedio de 3.56, esto significa que, de manera general, los empresarios estuvieron medianamente de acuerdo con que realizan acciones y prácticas de esas dimensiones de la RSE, lo que es considerado como aceptable; seguido por la dimensión ambiental e innovación con un promedio de 3.18 y 3.26 respectivamente, lo que se interpreta como no aceptable. 
RESPONSABILIDAD SOCIAL EMPRESARIAL E INNOVACIÓN COMO ESTRATEGIA COMPETITIVA EN EL SECTOR TURÍSTICO DE CIUDAD DEL CARMEN, CAMPECHE.

\section{Tabla 3}

Promedio de las dimensiones de responsabilidad social empresarial e innovación

\begin{tabular}{lc}
\hline Dimensiones & Promedio \\
\hline Dimensión Social & 3.60 \\
Dimensión económica & 3.56 \\
Dimensión ambiental & 3.18 \\
Innovación & 3.26 \\
\hline
\end{tabular}

Fuente: Elaboración propia a partir de los resultados.

Analizando de manera puntual cada una de las dimensiones de la variable RSE, se tiene los resultados siguientes.

\section{Dimensión social}

Con ella se buscaba medir el grado de participación que tienen las empresas observados en acciones a favor de la sociedad y con sus empleados. A partir de los datos obtenidos, se puede mencionar que fue la mejor evaluada; no obstante, hubo algunos ítems con resultados poco favorables. Al respecto, se pueden destacar como aceptables, aquellos relacionados con la contratación de personal en riesgo de exclusión, mejorar la calidad de vida de los empleados y considerar las propuestas de los empleados para tomar decisiones de gestión.

Se observa que los ítems con promedio más bajo, por lo tanto, considerados como no aceptables, están relacionados con los pagos de pensión para los empleados, aspectos del salario y la participación de las empresas en proyectos sociales.

Se puede apuntar que las empresas del sector turismo de Ciudad del Carmen, Campeche, no se preocupan por tener planes de pensiones para los empleados siendo ésta la menos valorada por las empresas ya que obtuvo un promedio de 3.24, como no aceptable. De igual forma, existe poca participación de las empresas en proyectos sociales ya que $13.45 \%$ de los encuestados estuvieron en desacuerdo; $18.49 \%$, en total desacuerdo y $1.68 \%$ mencionó no saber o no aplica. 
RESPONSABILIDAD SOCIAL EMPRESARIAL E INNOVACIÓN COMO ESTRATEGIA COMPETITIVA EN EL SECTOR TURÍSTICO DE CIUDAD DEL CARMEN, CAMPECHE.

\section{Dimensión económica}

Esta dimensión obtuvo un promedio general que la coloca en segundo lugar. Entre las actividades evaluadas con un valor mayor se encuentran: ofrecer calidad de los bienes y/o servicio; respetar los derechos de los consumidores; y proporcionar a los clientes información clara y completa, consideradas como aceptables. Por el contrario, entre los ítems con promedio más bajo y, por lo tanto, como no aceptables, destaca que las empresas carecen de una gestión merecedora de apoyo público regional o nacional, con un promedio de 3.18; además, con 3.24 se encuentran las acciones para fomentar una relación comercial con las empresas de la región. Así mismo, se puede mencionar que el $14.29 \%$ de los encuestados señalaron que no se ofrece una garantía más amplia que la media del sector.

\section{Dimensión ambiental}

En cuanto a la dimensión ambiental de la RSE, fue la que obtuvo menor promedio, que se puede interpretar como la de menor importancia para los empresarios, aunque tendría que ser todo lo contrario, pues Ciudad del Carmen, Campeche, se encuentra dentro del Área Natural Protegida Laguna de Términos (ANPLT).

Cabe señalar, que únicamente la planificación de las inversiones tomando en cuenta la reducción del impacto ambiental que generan, con una media de 3.50 y, estar a favor de la reducción de emisión de gases, residuos y reciclajes de materiales, el cual obtuvo una media de 3.71, pueden ser consideradas como aceptables acorde con la respuesta de los encuestados. La mayoría de las acciones se pueden catalogar como no aceptables en correspondencia a la escala usada, entre las que se encuentran la realización de auditorías ambientales; el sobrepaso voluntario de las regulaciones legales ambientales; y, por último, las participaciones en actividades relacionadas con la protección y mejora del entorno natural.

Finalmente, en concordancia con los administradores de las pymes objeto de estudio, un $60 \%$ de las empresas no realizan periódicamente auditorías ambientales, lo que denota la falta de importancia a realizar acciones de RSE de esta dimensión. Además, un $64.71 \%$ sobrepasan voluntariamente las regulaciones legales ambientales, mientras que el 35.29\% restante dicen si valorarlo. 
RESPONSABILIDAD SOCIAL EMPRESARIAL E INNOVACIÓN COMO ESTRATEGIA COMPETITIVA EN EL SECTOR TURÍSTICO DE CIUDAD DEL CARMEN, CAMPECHE.

\section{Innovación}

La variable innovación, en general, obtuvo un promedio 3.26, esto puede significar que no es relevante para las empresas del sector turismo de la ciudad adoptar una postura de innovación o simplemente ignoran como hacerlo, dado que es una calificación no aceptable. Dentro de los ítems considerados como aceptables están aquellos relacionados con: la intensificación del uso de las TIC, la cual obtuvo un promedio de 3.74; el potenciamiento de la presencia de la empresas en internet; y la formación interna y externa del personal con la finalidad de mejorar el conocimiento y el trabajo creativo de la empresa, el cual obtuvo un promedio de 3.53, lo que quiere decir que las empresas están fuertemente interesadas en la formación de su personal ya que lo identifican como mejora en la empresa.

Por el contrario, se encontraron varios ítems en esta variable con promedios bajos, de los cuales se pueden mencionar los referentes con introducir nuevas prácticas que potencien la captación de nuevos mercados nacionales, donde más del 50\% de los encuestados no valoran implementarlo; el segundo trata de igual manera de introducir nuevas prácticas que potencien la captación de nuevos mercados, esta vez internacionales a lo cual obtuvieron un promedio de 2.85 que se interpreta como no aceptable, es decir, las empresas no realizan acciones que les permitan captar un nuevo mercado nacional o internacional; y el tercer ítem más bajo trata de la implantación de nuevos productos y/o servicios, a lo cual menos del 50 \% empresas están interesadas en esta parte.

\section{CONCLUSIONES}

Se puede afirmar que, en cuanto al tamaño de las pymes de Ciudad del Carmen, Campeche participantes en esta investigación, un $75 \%$ fueron de empresas pequeñas y el $25 \%$ restante eran organizaciones medianas y el $68.07 \%$ eran empresas familiares.

De la revisión literaria se puede concluir que no existen muchos estudios que aborden ambos conceptos en su conjunto; sin embargo, se reconoce que los modelos de negocios turísticos que incorporan la RSE como estrategia de competitividad son de interés para la investigación académica, y que los autores coinciden al señalar que la RSE es una ventaja competitiva. Asimismo, se puede afirmar que, de acuerdo con Macías (2012), los vínculos entre la innovación y la RSE parten del beneficio social. 
RESPONSABILIDAD SOCIAL EMPRESARIAL E INNOVACIÓN COMO ESTRATEGIA COMPETITIVA EN EL SECTOR TURÍSTICO DE CIUDAD DEL CARMEN, CAMPECHE.

Los resultados observados, confirman que las pymes del sector turismo de Ciudad del Carmen, Campeche, están en una situación aceptable según las acciones de RSE que señalaron sus administradores realizar. En cuanto a la innovación, de acuerdo con los señalamientos de PérezAranda (2015), es necesario diversificar la oferta local con nuevas prácticas que potencien la captación de nuevos clientes ante la posibilidad de la apertura de un nuevo mercado nacional o internacional, que para Ciudad del Carmen, Campeche responda a la industria petrolera y al turismo recreativo.

Por otro lado, se puede concluir la importancia de las acciones de RSE como elemento de la innovación empresarial, factor de crecimiento ya que, en el mercado globalizado, estas le proporcionan una ventaja competitiva a largo plazo. Acciones necesarias ante los cambios que se vislumbran tanto en el sector petrolero, como los derivados por la pandemia por Covid-19.

Finalmente, como futura línea de investigación, es necesario medir la relación entre la RSE, con base en sus dimensiones, y la innovación, en el marco de la competitividad de este sector, determinando la causalidad de la relación entre las variables observadas. Pues, con ello estaría encontrando una explicación de la innovación a partir de cada una de las dimensiones y los respectivos indicadores de la RS en el contexto actual de la pandemia, lo que permitiría orientar futuros trabajos académicos que sirvieran de apoyo a las organizaciones que pretendan mejorar en RSE.

Por último, una recomendación metodológica para investigaciones posteriores: evitar los errores en la aplicación del instrumento, buscando con ello mantener el tamaño de la muestra calculado estadísticamente a partir de un margen de error no mayor al $5 \%$.

\section{REFERENCIAS BIBLIOGRÁFICAS}

Behar, D. (2008). Introducción a la metodología de la investigación. Shalom.

Bermúdez, K. (2007). La RSE y los negocios. Universidad Autónoma de México.

Bowen, H. (1953). Social responsabilities of the businessman. New York: Harper \& Row.

Cajiga, J. (2017). El concepto de responsabilidad social empresarial. Centro Mexicano para la Filantropía, CEMEFI, México. 
RESPONSABILIDAD SOCIAL EMPRESARIAL E INNOVACIÓN COMO ESTRATEGIA COMPETITIVA EN EL SECTOR TURÍSTICO DE CIUDAD DEL CARMEN, CAMPECHE.

Carroll, A. (1991). The pyramid of corporate social responsibility: toward the moral management of organizational stakeholders. Bussiness horizons, 39-48.

Comisión de las Comunidades Europeas [CCE]. (2001). Libro verde: fomentar un marco europeo para la responsabilidad social de las empresas. Bruselas: Comisión de las Comunidades Europeas. https://sid.usal.es/idocs/F8/FDO11925/libroverde.pdf

Dahlsrud, A. (2008). How corporate social responsibility is defined: an analysis of 37 definitions, corporate social responsibility and environmental management.

Davis, K. (1967). Understanding the social responsability puzzle. Bussines Horizons, 10, 45-51.

Davis, K. (1973). The Case for and against Business Assumption of Social Responsibilities. The Academy of Management Journal, 312-322.

Drucker, P. (1984). The new meaning of corporate social responsabillity. California management Review, 53-63.

Flores, J., Ogliastri, E., Peinado-Vara, E. \& Petry, E. (2007). El argumento empresarial de la RSE: 9 casos de América Latina y el Caribe. Washington: Banco Interamericano de Desarrollo.

Frías, D. (2019). Apuntes de consistencia interna de las puntuaciones de un instrumento de medida. España: Universidad de Valencia. https://www.uv.es/friasnav/AlfaCronbach.pdf

Friedman, M. (1962). Capitalism and Freedom. Chicago University Press.

Friedman, M. (1970). The social responsibillity of business is to increase its profits. The New York Times Magazine, 1-6.

Gallardo-Vázquez, D. \& Sánchez-Hernández, I. (2013). Análisis de la incidencia de la Responsabilidad Social Empresarial en el éxito competitivo de las microempresas y el papel de la innovación, Universia Bussines Review, 14-31.

Gallardo-Vázquez, D., Sánchez-Hernández, I. \& Corchuelo-Martínez-Azúa, M. (2013). Validación de un instrumento de medida para la relación entre la orientación a la responsabilidad social corporativa y otras variables estratégicas de la empresa. Revista de Contabilidad - Spanish Accounting Review, 16(1) (2013), 11-23.

Garriga, E. y Melé, D. (2004). Corporate social responsability theories: mapping the territory. Journal of Business Ethics, 51-71.

Global Reporting Initiative (GRI). (s.f.). La elaboración de memorias de sostenibilidad de GRI: ¿vale la pena el viaje?

Hernández, R., Fernández, C., Baptista, P., Méndez, S. y Mendoza, C. (2014). Metodología de la investigación. México, D.F.: McGraw-Hill.

Hidalgo, A., León, G. \& Pavón, J. (2002). La Gestión de la Innovación y la Tecnología en las Organizaciones. Madrid: Editorial Pirámide.

Instituto Ethos. (2005). Indicadores Ethos de responsabilidad social. Instituto Ethos. Recuperado de http://www.centroetica.uct.cl/documentos/archivos/PDF/H1\%2007.pdf 
RESPONSABILIDAD SOCIAL EMPRESARIAL E INNOVACIÓN COMO ESTRATEGIA COMPETITIVA EN EL SECTOR TURÍSTICO DE CIUDAD DEL CARMEN, CAMPECHE.

Instituto Nacional de Estadística y Geografía [INEGI]. (2014). Recuperado de https://www.inegi.org.mx/datos/

International Organization for Standardization (ISO). (2010). ISO 26000 Social Responsibility. ISO. Recuperado de https://www.iso.org/iso-26000-social-responsibility.html

Kirchhoff, S. (2000). Green Business and Blue Angels: A Model of Voluntary Overcompliance with Asymetric Information. Environmental \& Resource Economics, 15, 403-420.

López-Noriega, M., Zalthen, L. \& Cervantes, M. (2019). Análisis comparativo de la competitividad en el sector hotelero en estados de los dos golfos de México. Revista de Investigación Latinoamericana en Competitividad Organizacional, RILCO, (1). https://www.eumed.net/rev/rilco/01/sector-hotelero.html

Macías, H. (2012). Innovación y responsabilidad social: una reflexión sobre los puntos de encuentro. Revista Universidad y Empresa, 13(21), 13-35.

McGuire, J. (1963). Bussines and society. New york: McGraw-Hill Interamericana.

Mercado, H. y Palmerín, M. (2012). El turismo y su impacto en la economía de México y del estado de Michoacán. TURyDES, 5(12).

Nieto, M. \& Fernández, R. (2004). Responsabilidad social corporativa: la última innovación del management. Universia Business Review, Primer trimestre, 28-39.

OCDE. (2005). Manual de Oslo. Guía para la recogida e interpretación de datos de innovación. OECD/European Communities,

OCDE. (2019), OECD SME and Entrepreneurship Outlook 2019, OECD Publishing, Paris, https://doi.org/10.1787/34907e9c-en.

Pérez, H. (2012). Metodología de la investigación. Colegio militar de la nación.

Pérez-Aranda, J. (2015). La responsabilidad social corporativa en turismo. Estado de la cuestión. ARA Revista de Investigación en Turismo, 5(1), 63-81

Porter, M. \& Kramer, M. (2002). La responsabilidad social de la empresa: evolución de conceptos y prácticas en América Latina y el Caribe. En: Flores, J., Ogliastri, E., Peinado-Vara, E. \& Petry, E. (2007). El argumento empresarial de la RSE: 9 casos de América Latina y el Caribe. Washington: Banco Interamericano de Desarrollo.

Porter, M. \& Kramer, M. (2006). Estrategia y sociedad. Harvard Business Review, 42-56.

Scarone, C. (2005). La innovación en la empresa: la orientación al mercado como factor de éxito en el proceso de innovación en producto. (Tesis doctoral). Internet Interdisciplinary Institute (IN3) - Universitat Oberta de Catalunya (UOC), Cataluña. http://www.uoc.edu/in3/dt/esp/scarone0405.html

Schroeder, K. \& Kilian, B. (2007). El efecto de las prácticas de RSE en los Ingresos de los Negocios. Washington: Banco Interamericano de Desarrollo.

Schumpeter, J. A. (2002). The theory of economic development. Harvard University Press. 
RESPONSABILIDAD SOCIAL EMPRESARIAL E INNOVACIÓN COMO ESTRATEGIA COMPETITIVA EN EL SECTOR TURÍSTICO DE CIUDAD DEL CARMEN, CAMPECHE.

Shapiro, C. (1983). Premiums for High Quality Products as Returns to Reputations. Quarterly Journal of Economics, 98(4), 659-679.

Toro, D. (2006). El enfoque estratégico de la responsabilidad social corporative. Intangible capital, 14(2), 338-358.

Turban, D. \& Greening, D. (1997). Corporate Social Performance and Organizational Attractiveness to Prospective Employees. Academy of Management Journal, 40(3), 658673.

Wood, D. (1991). Corporate social performance revised. Academy of Management Review, 691718.

World Travel \& Tourism Council (2016). World travel \& tourism council. Recuperado de https://wttc.org/ 